\title{
Diffraction of swift atoms after grazing scattering from metal surfaces: N/Ag(111) system
}

\author{
M. S. Gravielle, ${ }^{1, *}$ G. A. Bocan,,$^{2, \dagger}$ and R. Díez Muiño ${ }^{3}$ \\ ${ }^{1}$ Instituto de Astronomía y Física del Espacio (CONICET-UBA) and Departamento de Física, Facultad de Ciencias Exactas y Naturales, \\ Universidad de Buenos Aires, Buenos Aires, Argentina \\ ${ }^{2}$ Centro Atómico Bariloche, Comisión Nacional de Energía Atómica, and Consejo Nacional de Investigaciones Científicas y Técnicas, \\ S.C. de Bariloche, Río Negro, Argentina \\ ${ }^{3}$ Donostia International Physics Center (DIPC) and Centro de Física de Materiales CSIC-UPV/EHU, San Sebastián, Spain
}

(Received 16 July 2010; published 11 November 2010)

\begin{abstract}
Diffraction patterns produced by grazing scattering of fast $\mathrm{N}$ atoms from a $\mathrm{Ag}(111)$ surface are investigated by employing the surface eikonal approximation. This method is a distorted-wave theory that takes into account the coherent addition of contributions coming from different projectile paths. In the model the projectile-surface potential is obtained from an accurate density-functional theory calculation. The dependence of the scattered projectile spectra on impact energy and incidence channel is analyzed, and possible incident direction and energy range for the observation of the interference patterns are predicted. In addition, it is found that as a result of the high reactivity of $\mathrm{N}$ atoms, asymmetries of the surface potential might be detected through their effects on diffraction patterns.
\end{abstract}

DOI: 10.1103/PhysRevA.82.052904

PACS number(s): 79.20.Rf, 79.60.Bm, 34.20.Cf, 61.85.+p

\section{INTRODUCTION}

Diffraction of atoms and molecules in the keV energy range due to grazing scattering from crystal surfaces is attracting considerable attention nowadays [1-4]. The interest is motivated by two different factors: (i) the fast velocity of incident projectiles, which made the observation of interference effects an unexpected phenomenon [5-7], and (ii) the extreme sensitivity of the scattering distributions to the projectile-surface interaction [8-11], which opens the way for the development of a powerful surface analysis technique.

The first experimental evidence of this diffraction effect was reported at insulator surfaces [5,6]. It was argued that the movement of the projectile along a certain channeling direction (too fast to allow for diffraction) could somehow be decoupled from its much slower motion within the plane perpendicular to it, and diffraction patterns were thus originated by this transverse motion. Insulator surfaces provided a favorable setting as the presence of a band gap strongly suppressed inelastic electronic processes, preventing quantum decoherence. However, diffraction for fast atoms in grazing incidence has recently been observed at metallic materials as well [12-14], although electron excitations were expected to smudge quantum interference signatures.

As it is still in an explorative phase, fast-atom diffraction from metal surfaces is not yet fully understood. Experimental works [2,12-14] on the subject were carried out for different metallic surfaces, such as $\mathrm{Ag}, \mathrm{Ni}$, or even Fe with adsorbed $\mathrm{S}$ or $\mathrm{O}$ atoms, but the projectiles were in contrast restricted to only light nonreactive ones, mostly $\mathrm{He}$ atoms. The goal of this article is to study the influence of the projectile on the diffraction process by considering the scattering of open-shell nitrogen atoms on a $\operatorname{Ag}(111)$ surface. This collision

\footnotetext{
*msilvia@iafe.uba.ar

${ }^{\dagger}$ Donostia international Physics Center (DIPC), Donostia/San Sabastián, Spain.
}

system displays a stronger and much more position-dependent interaction [15] than that of He-Ag.

To describe the diffraction process, we employ a distortedwave model - the surface eikonal approximation [10] - that makes use of the eikonal wave function to represent the elastic collision with the surface, while the movement of the fast projectile is described classically by considering axially channeled trajectories for different initial conditions. The surface eikonal approximation is valid for small de Broglie wavelengths of incident atoms, as considered here, which are several orders of magnitude smaller than the interatomic spacings in the crystal. This method has already been applied to investigation of fast-atom diffraction from insulator surfaces [11], providing results in good agreement with experimental data [3].

A key point in the description of the diffraction patterns is the detailed representation of the projectile-surface potential. In previous articles [12,14], diffraction from Ag surfaces was described within the hard-wall approximation, modeling the projectile-surface interaction as a two-dimensional sinusoidal function that represented the averaged potential along the incidence direction. In order to allow for an accurate description of the $\mathrm{N}-\operatorname{Ag}(111)$ interaction, we have used instead a full three-dimensional (3D) potential energy surface (PES), obtained from density-functional theory (DFT) calculations, which gives the complete dependence of the system's energy on the projectile's three degrees of freedom. Multidimensional PESs have proven to be useful tools for studying the dynamics of atoms or diatomic molecules impinging on metallic surfaces [16-20]. In our case, we have used the VIENNA AB INITIO SIMULATION PROGRAM (VASP) code [21] in combination with an elaborate interpolation technique [22] to evaluate the PES for the $\mathrm{N} / \mathrm{Ag}(111)$ system.

We calculate angular distributions of $\mathrm{N}$ projectiles that impact on a $\operatorname{Ag}(111)$ surface along different low-index crystallographic directions and analyze how these distributions are affected by the characteristics of the considered channel. The role played by inelastic collision processes associated with electronic excitations is also investigated. This article is 
organized as follows: The theoretical formalism is summarized in Sec. II; results are presented and discussed in Sec. III; and we outline our conclusions in Sec. IV. Atomic units (a.u.) are used unless otherwise stated.

\section{THEORETICAL MODEL}

\section{A. The transition amplitude}

Within the surface eikonal model, the scattering state of the projectile, $\Psi_{i}^{+}$, is approximated by the eikonal-Maslov wave function [3], which reads

$$
\Psi_{i}^{+}\left(\vec{R}_{P}, t\right) \simeq \Phi_{i}\left(\vec{R}_{P}\right) \exp [-i \eta(t)]
$$

where $\vec{R}_{P}$ denotes the position of the incident atom, $\eta$ is the eikonal-Maslov phase, and $\Phi_{i}\left(\vec{R}_{P}\right)=(2 \pi)^{-3 / 2} \exp \left(i \vec{K}_{i} \cdot \vec{R}_{P}\right)$ is the initial unperturbed wave function, with $\vec{K}_{i}$ as the initial projectile momentum. The phase $\eta$ depends on the classical position of the incident atom at the time $t, \overrightarrow{\mathcal{R}}_{P}(t)$, as

$$
\eta(t)=\int_{-\infty}^{t} d t^{\prime} V_{S P}\left(\overrightarrow{\mathcal{R}}_{P}\left(t^{\prime}\right)\right)+\phi_{M},
$$

where $V_{S P}$ is the projectile-surface interaction and $\phi_{M}=\nu \pi / 2$ is the Maslov correction term that takes into account the phase change suffered by a wave as it passes through a focus, with $v$ the Maslov index defined as in Ref. [23].

By replacing Eq. (1) in the usual definition of the transition matrix [24], after some steps of algebra the eikonal $T$-matrix element reads [10]

$$
T_{i f}^{\mathrm{eik}}=\int d \vec{R}_{o s} a_{i f}\left(\vec{R}_{o s}\right)
$$

where $\vec{R}_{o s}$ determines the initial position of the projectile on the surface plane and

$$
\begin{aligned}
a_{i f}\left(\vec{R}_{o s}\right)= & \frac{1}{(2 \pi)^{3}} \int_{-\infty}^{+\infty} d t\left|v_{z}\left(\overrightarrow{\mathcal{R}}_{P}\right)\right| \\
& \times \exp \left[-i \vec{Q} \cdot \overrightarrow{\mathcal{R}}_{P}-i \eta\left(\overrightarrow{\mathcal{R}}_{P}\right)\right] V_{S P}\left(\overrightarrow{\mathcal{R}}_{P}\right)
\end{aligned}
$$

is the transition amplitude associated with the classical path $\overrightarrow{\mathcal{R}}_{P}\left(\vec{R}_{o s}, t\right)$. In Eq. (4) $\vec{Q}=\vec{K}_{f}-\vec{K}_{i}$ is the projectile momentum transfer, with $\vec{K}_{f}$, the final projectile momentum, being $\left|\vec{K}_{f}\right|=\left|\vec{K}_{i}\right|$. The function $v_{z}\left(\overrightarrow{\mathcal{R}}_{P}\right)$ denotes the component of the projectile velocity perpendicular to the surface plane, with $\hat{z}$ directly along the surface normal, toward the vacuum region. A schematic representation of the process and the coordinate system is displayed in Fig. 1. Details of the calculation are given in Refs. [10,11].

\section{B. Projectile-surface interaction}

The interaction energy of the $\mathrm{N}$ atom with the $\operatorname{Ag}(111)$ surface is described here with a full adiabatic 3D PES that depends on the atomic position $\vec{R}_{P}=(X, Y, Z)$. The PES is constructed from a grid of $615 a b$ initio energies over which an interpolation is performed. All $a b$ initio data are obtained from the DFT-based VASP code [21], which uses a plane-wave basis set to expand the system wave functions and is particularly efficient to model metallic surfaces. The values of relevant VASP parameters are chosen so that $a b$ initio energies are

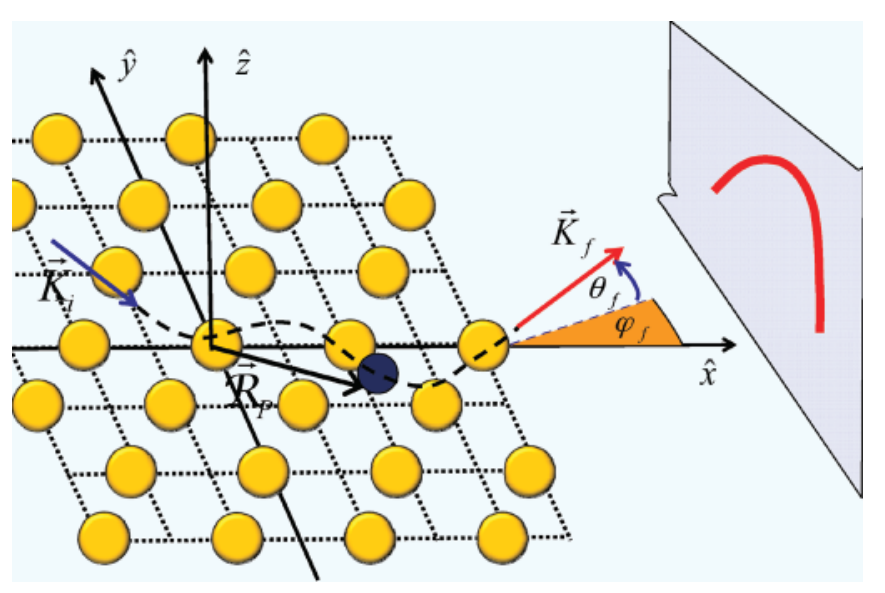

FIG. 1. (Color online) Schematic depiction of the elastic collision process and the coordinate system.

calculated to a prescribed accuracy. The exchange-correlation energy is calculated within the generalized gradient approximation (GGA) and using the Perdew-Wang energy functional (PW91) [25]. The electron-core interaction is described with ultrasoft pseudopotentials [26]. The energy cutoff in the plane-wave expansion is $452.526 \mathrm{eV}$, corresponding to a high-precision VASP calculation; the fractional occupancies are determined through the broadening approach of Methfessel and Paxton [27] with $N=1$ and $\sigma=0.2$; and the Brillouinzone integration is performed with a $5 \times 5 \times 1 \gamma$-centered grid of special $k$ points.

The Ag lattice constant, obtained from a bulk calculation, is $a=4.17 \AA$. The $\operatorname{Ag}(111)$ surface is modeled by means of the supercell-slab scheme. A five-layer slab is used with a $2 \times 2$ cell in the plane parallel to the surface (atomic coverage of 0.25 ) and a supercell of length $24.08 \AA$ along the normal to the surface ( $\hat{z}$ axis). The latter length guarantees that the energy for $\mathrm{N} / \operatorname{Ag}(111)$, with the $\mathrm{N}$ atom midway between slabs, is $X Y$-independent and thus provides a reasonable representation of the asymptotic region. This configuration, for which there is hardly any interaction with the surface, is chosen as the energy reference.

In order to get the surface equilibrium geometry, the interlayer distance is relaxed from its bulk value $d_{0}=2.408 \AA$. The third layer is kept fixed during the relaxation process, which continues until the difference in energy between consecutive iterations is less than $1 \mathrm{meV}$. Geometry corrections due to surface relaxation are, however, almost negligible. The first layer moves into the vacuum so that the relaxed first interlayer distance is $d_{12} / d_{0}=1.0009$. The second layer moves into the bulk so that $d_{23} / d_{0}=0.9956$. Once relaxed, the slab is kept frozen for the calculations that follow.

Given the open-shell electronic structure of the $\mathrm{N}$ atom $\left(1 s^{2} 2 s^{2} 2 p^{3}\right)$, an adequate description of the ground state requires a spin-polarized calculation. A 3D energy grid is obtained, consisting of 41 equidistant points $Z$ in the range $-1.5 \AA \leqslant Z \leqslant 6.5 \AA$, with $Z=0$ corresponding to the topmost surface layer and 15 sites $[(X, Y)$ values] uniformly spread within the unit cell. Once the 615-point energy grid is derived, the PES is built by interpolating over the $a b$ initio data with the corrugation reducing procedure [22]. As a quality check of the interpolation, the output of the constructed 3D 
PES was contrasted to a set of $a b$ initio off-grid values and, for $Z>0.7 \AA$, differences with the $a b$ initio data were found to be lower than $5 \mathrm{meV}$.

\section{RESULTS}

We use the surface eikonal approximation to study angular distributions of neutral $\mathrm{N}$ atoms elastically scattered from a $\operatorname{Ag}(111)$ surface under axial grazing incidence conditions. Within the surface eikonal approach, the differential probability, per unit of surface area, for elastic scattering with final momentum $\vec{K}_{f}$ in the direction of the solid angle $\Omega_{f}$ is expressed as $d P / d \Omega_{f}=(2 \pi)^{4} m_{P}^{2}\left|\tilde{T}_{i f}^{\text {eik }}\right|^{2}$, where $m_{P}$ is the projectile mass and $\tilde{T}_{i f}^{\text {eik }}$ denotes the eikonal $T$-matrix element given by Eq. (3), normalized per unit area. As our interest lies in analyzing the effects of the corrugation of the projectile-surface interaction on the diffraction patterns, we have evaluated $T_{i f}^{\text {eik }}$ from Eq. (3) using $4 \times 10^{5}$ classical trajectories with random initial positions $\vec{R}_{o s}$ that vary within a reduced unit cell. This provides information on the shape of the potential through supernumerary rainbows $[3,7]$.

Two different incidence channels are considered in this work: $\langle\overline{1}, 0,1\rangle$ and $\langle\overline{1}, \overline{1}, 2\rangle$, which are equivalent to the $\langle\overline{1}, 1,0\rangle$ and $\langle\overline{2}, 1,1\rangle$ channels, respectively. For impact along these low-index crystallographic directions, we found that, as was the case for insulators [5-7,10], almost all classically scattered projectiles end on a semicircle of radius $\theta_{i}$, satisfying the relation $\theta_{f}^{2}+\varphi_{f}^{2} \approx \theta_{i}^{2}$, where $\theta_{i}$ is the incidence angle measured with respect to the surface plane. The final angles $\theta_{f}$ and $\varphi_{f}$ are the polar and azimuthal angles, respectively, determined by the final momentum $\vec{K}_{f}$, with $\varphi_{f}$ measured with respect to the $\hat{x}$ axis along the incidence direction in the surface plane, as depicted in Fig. 1.

We first consider incidence along the narrowest channel $\langle\overline{1}, \overline{1}, 2\rangle$, the effective width of which is 2.79 a.u.. In Fig. 2 the differential probability $d P / d \varphi_{f}$ is plotted, as a function of $\varphi_{f}$, for $3-\mathrm{keV} \mathrm{N}$ atoms impinging on the surface along the $\langle\overline{1}, \overline{1}, 2\rangle$ direction with a glancing angle $\left(\theta_{i}=0.47^{\circ}\right)$. In agreement with experimental findings for He projectiles [12,13], this angular distribution, originated by the scattering of $\mathrm{N}$ atoms from the $\operatorname{Ag}(111)$ surface, displays an oscillatory structure, with maxima and minima symmetrically placed with respect to the incidence direction, that corresponds to $\varphi_{f}=0$. Such an interference pattern is produced by the coherent addition of the contributions coming from nitrogen atoms that follow different paths but end scattered with the same final momentum. The outermost peaks of the spectrum of Fig. 2 are associated with rainbow scattering and can be classically explained. Notice that the extreme sharpness of these rainbow maxima is a consequence of the semiclassical description of the projectile motion, which does not include the decaying intensity on the dark side of the classical rainbow. However, a more elaborate quantum treatment of rainbow scattering [28] is not expected to affect the maxima arising between the outermost peaks, which are related to supernumerary rainbows $[3,7]$.

In order to analyze the dependence on the incidence energy $E_{i}$, we have split it into two terms: $E_{i}=E_{i \|}+E_{i \perp}$, where $E_{i \|}=E_{i} \cos ^{2} \theta_{i}\left(E_{i \perp}=E_{i} \sin ^{2} \theta_{i}\right)$ is associated with the component of the initial velocity parallel (perpendicular)

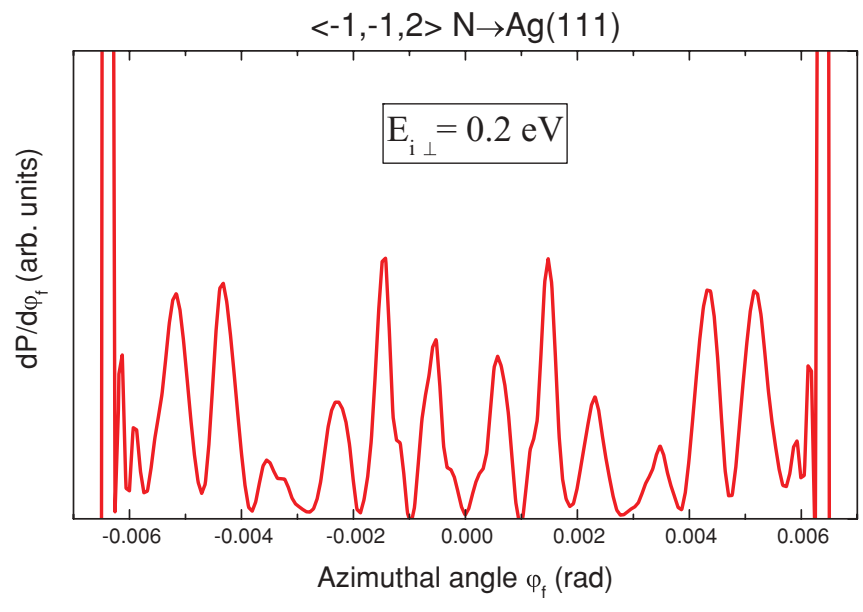

FIG. 2. (Color online) Azimuthal angular distribution of elastic scattered projectiles for 3-keV $\mathrm{N}$ atoms impinging on $\mathrm{Ag}(111)$ along the direction $\langle\overline{1}, \overline{1}, 2\rangle$, with the incidence angle $\theta_{i}=0.47^{\circ}$. The perpendicular energy, evaluated as explained in the text, is $0.2 \mathrm{eV}$.

to the axial channel. The preceding figure (Fig. 2) corresponds to a perpendicular energy $E_{i \perp}=0.2 \mathrm{eV}$. In Fig. 3 we plot the differential scattering probability as a function of the deflection angle $\Theta$, defined as $\Theta=\arctan \left(\varphi_{f} / \theta_{f}\right)$, for a higher perpendicular energy, $E_{i \perp}=0.5 \mathrm{eV}$. Two different total energies, $E_{i}=3$ and $7 \mathrm{keV}$, are considered. The number of interference maxima, as well as their $\Theta$ positions, are independent of $E_{i}$ for constant $E_{i \perp}$, as it had previously been observed for insulator surfaces [3]. In contrast to that case, however, we find that the relative intensities of intermediate maxima depend on the total energy $E_{i}$, some of them being higher for the lower incidence energy. This effect might be associated with the reactivity of $\mathrm{N}$ atoms, which originates a strong corrugation of the potential that affects the projectile movement along the incidence channel as the parallel velocity decreases.

When the perpendicular energy increases, the diffraction pattern becomes more complicated, presenting complex

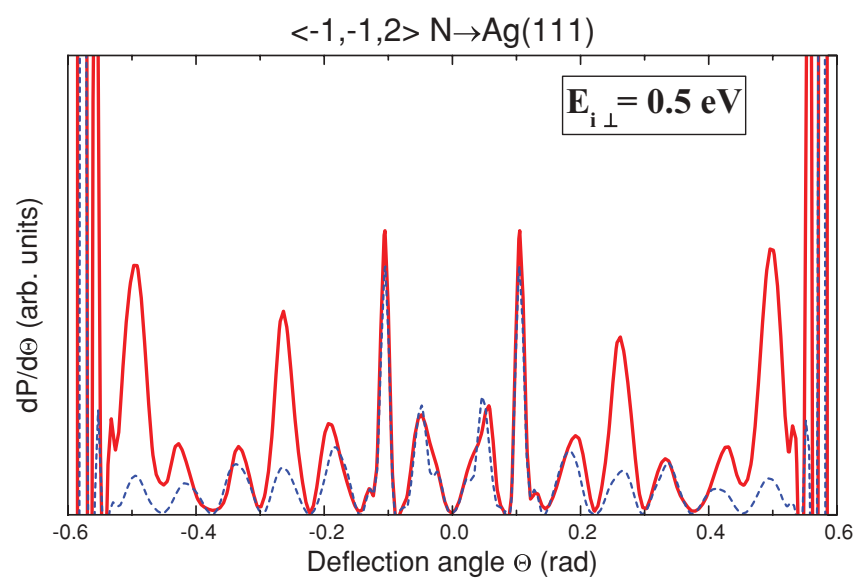

FIG. 3. (Color online) Angular projectile distributions, as function of the deflection angle $\Theta$, for $\mathrm{N}$ atoms scattered from $\operatorname{Ag}(111)$ along the direction $\langle\overline{1}, \overline{1}, 2\rangle$, with $E_{i \perp}=0.5 \mathrm{eV}$. Two different incidence energies are considered: solid line, $E_{i}=3.0 \mathrm{keV}$; and dashed line, $E_{i}=7.0 \mathrm{keV}$. 


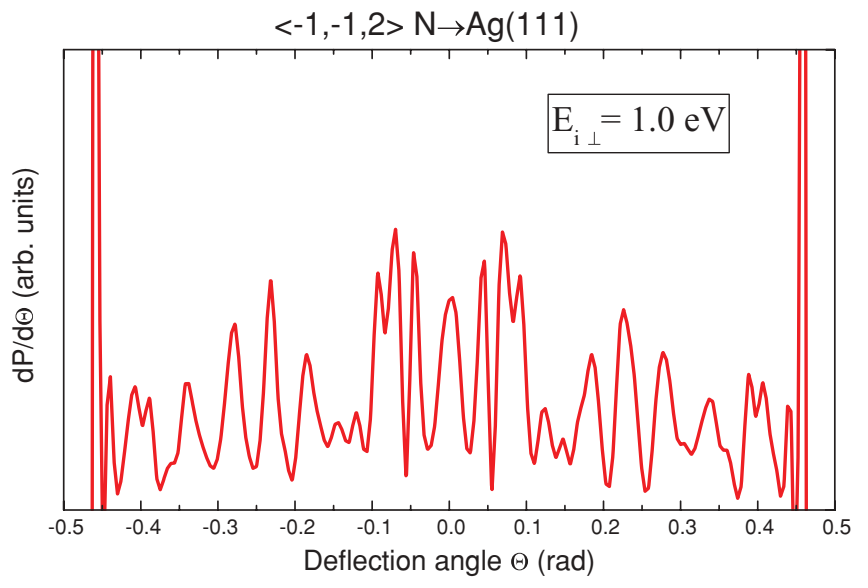

FIG. 4. (Color online) Similar to Fig. 3 for $3-\mathrm{keV} \mathrm{N}$ atoms impinging with the perpendicular energy $E_{i \perp}=1.0 \mathrm{eV}$.

interference structures, as shown in Fig. 4 for $E_{i \perp}=1 \mathrm{eV}$. Therefore, the energy range where regular supernumerary maxima can be observed is smaller than the one for helium impact on insulator surfaces [3]. This is a consequence of both the large mass of the projectile and the interaction with the metal surface, which tends to smudge interference effects, as shown by the experiments [13].

Something similar occurs when we consider scattering along the wider channel $\langle\overline{1}, 0,1\rangle$, whose effective width is 4.83 a.u.. For perpendicular energies lower than $1.5 \mathrm{eV}$, this channel presents an effective corrugation $\Delta z$ [29] seven times larger than that of the $\langle\overline{1}, \overline{1}, 2\rangle$ channel, that is, $\Delta z \simeq 0.08$ and $0.6 \AA$ for the $\langle\overline{1}, \overline{1}, 2\rangle$ and $\langle\overline{1}, 0,1\rangle$ channels, respectively, and both corrugations are nearly constant in this energy range. Results for incidence in the direction $\langle\overline{1}, 0,1\rangle$ with a perpendicular energy $E_{i \perp}=0.2 \mathrm{eV}$ are displayed in Fig. 5 . We observe that even for this low perpendicular energy the angular distribution presents a complex pattern, indicating that this channel provides a narrower window to observe diffraction effects as interference signatures start to blur when the width and corrugation of the channel augment. In addition, the right-left symmetry with respect to the deflection angle $\Theta=0$ is lost in Fig. 5, due to the asymmetry of the PES corrugation across the $\langle\overline{1}, 0,1\rangle$ channel. This asymmetry is related to the $\mathrm{ABCABC} \ldots$ stacking of the $\operatorname{Ag}(111)$ surface, shown in Fig. 6(a). Four unit cells, containing the $X Y$ grid based on 15 irreducible sites, are displayed. The Ag atoms of the first layer correspond to the sites labeled TOP, while points labeled HCP (hexagonal close-packed) and FCC (face-centered cubic) stand on top of $\mathrm{Ag}$ atoms of the second and third layers, respectively. The inequivalence between points in triangles pointing up and down results in energy differences, which are presented in Fig. 6(b) for the characteristic case of the HCP and FCC sites. Note that even though right-left asymmetries also exist across some channels considered for $\mathrm{He} / \mathrm{Ag}(110)$ [12], in our system this is reinforced by reactive $\mathrm{N}$ atoms resulting in an accentuated position dependence of the system's PES.

Finally, note that although inelastic processes are supposed to provide an important source of decoherence for the case of metal surfaces, our treatment only describes the elastic collision channel. We have therefore investigated the inelastic

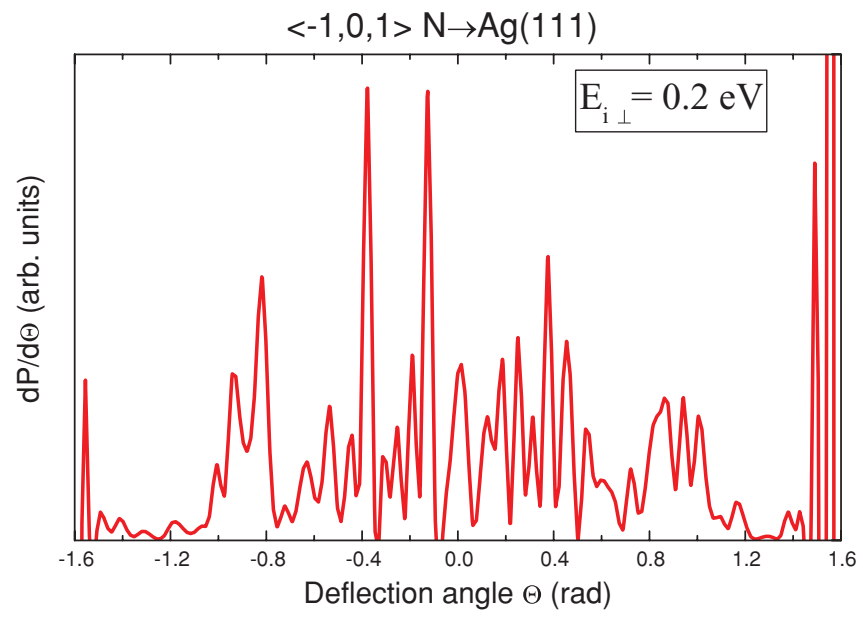

FIG. 5. (Color online) Similar to Fig. 4 for scattering along the direction $\langle\overline{1}, 0,1\rangle$, with $E_{i \perp}=0.2 \mathrm{eV}$.

scattering probability for the $\mathrm{N} / \mathrm{Ag}(111)$ system. Contributions due to inelastic collisions of the projectile with target atomic nuclei or core electrons are both negligible in the considered energy range [30]. Then, we assume that inelastic processes are mainly associated with excitations of valence band electrons. For a projectile that moves parallel to the surface at given distance $Z$ with a high velocity $\vec{v}$, we evaluate
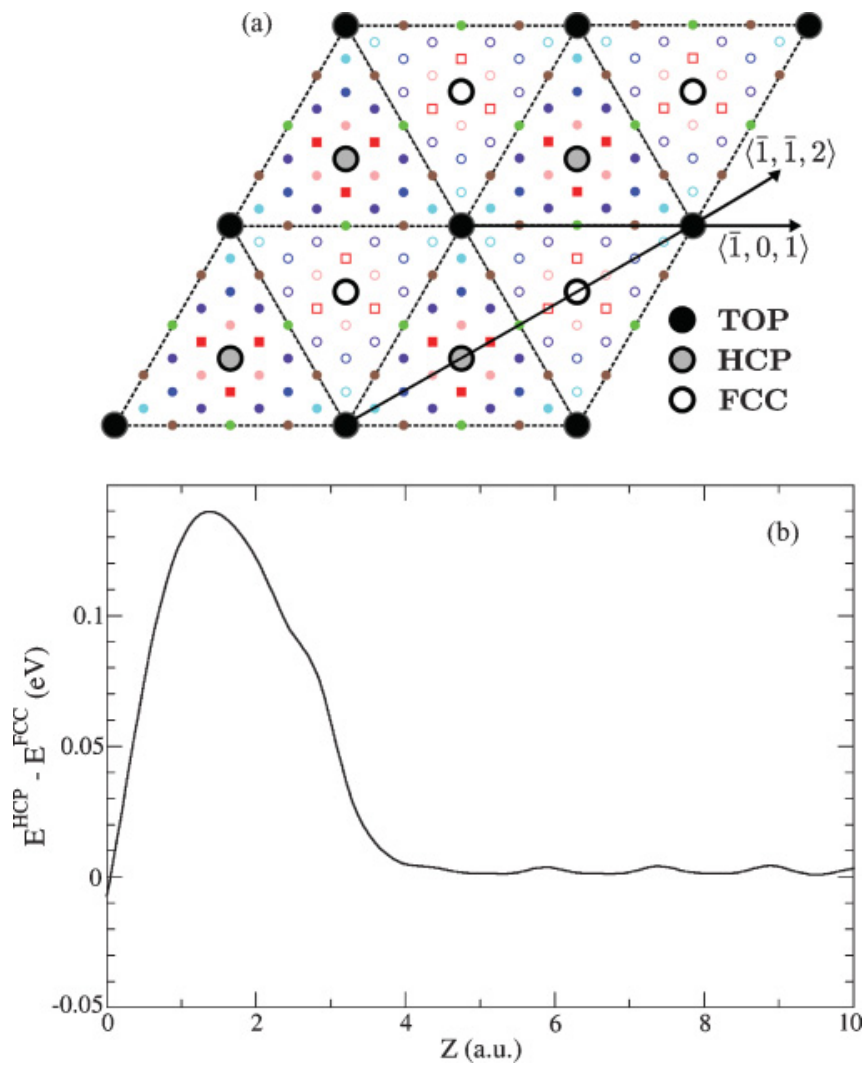

FIG. 6. (Color online) (a) Surface geometry and symmetry. Symbols indicate the $X Y$ grid, with TOP, HCP, and FCC points placed on top of Ag atoms of the first, second, and third layers, respectively. (b) Energy difference between PES values at HCP and FCC positions, as a function of the distance $Z$ to the topmost atomic layer. 
the differential probability of electron excitations per unit path length by means of the usual dielectric formalism [31]. It reads

$$
\frac{d P_{\mathrm{in}}}{d X}(Z)=-\int_{0}^{+\infty} \frac{d \omega}{\pi v^{2}} \int_{\omega / v}^{+\infty} d q_{\|} z_{P}^{2}\left(q_{\|}\right) \frac{\operatorname{Im}\left[\phi_{\text {ind }}\left(q_{\|}, \omega, Z\right)\right]}{\sqrt{q_{\|}^{2}-(\omega / v)^{2}}}
$$

where $\vec{q}_{\|}$is the transferred momentum parallel to the surface and $\omega=\vec{q}_{\|} \cdot \vec{v}$ is the transferred energy. The function $\phi_{\text {ind }}$ is the Fourier transform of the surface potential induced by a bare projectile, divided by the corresponding projectile potential, and it is represented here within the specular reflection model [32]. The function $z_{P}\left(\vec{q}_{\|}\right)$denotes the effective projectile charge, defined as in Ref. [33], which takes into account the screening of the projectile nuclear charge produced by atomic electrons.

The mean value of the total probability of electronic transitions was obtained from Eq. (5) by integrating along the different axially channeled trajectories. For $3-\mathrm{keV} \mathrm{N}$ atoms impinging along the $\langle\overline{1}, \overline{1}, 2\rangle$ direction, we found inelastic transition probabilities that lead to values of the mean-free path (MFP) that decrease with increasing $E_{i \perp}$. For $E_{i \perp}$ in the range $0.2-1.0 \mathrm{eV}$, the MFPs obtained are in the corresponding 130-70 a.u. interval. The characteristic distance of the scattering events, which also decreases as a function of $E_{i \perp}$, is between 1,000 and 550 a.u.. Given these values, inelastic scattering processes do not seem to be relevant for the perpendicular energies considered in this work since, on average, projectiles suffer less than eight inelastic collisions along the whole path. We should point out, on the one hand, that the inclusion of surface band structure effects might modify our estimation for the contribution of electronic excitations [34,35]. On the other hand, interference patterns have been experimentally observed for the $\mathrm{He} / \mathrm{Ag}(110)$ system [12], with similar incidence conditions and a measured energy loss that is fairly close to the one we obtain for our system (about $14 \mathrm{eV}$ ).

\section{CONCLUSIONS}

We have studied elastic scattering of swift $\mathrm{N}$ atoms from a metal $\operatorname{Ag}(111)$ surface under axial grazing incidence conditions. The collision process has been described by means of the surface eikonal approximation, which takes into account the quantum interference originated by the coherent superposition of transition amplitudes corresponding to different projectile paths. The projectile-surface interaction was obtained by combining ab initio calculations and an elaborate interpolation technique. The derived 3D PES provides an accurate description of the surface potential.

Angular distributions of $\mathrm{N}$ atoms scattered from the $\operatorname{Ag}(111)$ surface display diffraction patterns that depend on the axial incidence direction. For scattering along the $\langle\overline{1}, \overline{1}, 2\rangle$ channel, the angular spectrum presents interference structures, with peaks symmetrically placed with respect to the incidence direction. The complexity of these structures augments dramatically as $E_{i \perp}$ increases up to $1.0 \mathrm{eV}$, indicating that the scattering of $\mathrm{N}$ atoms from a $\mathrm{Ag}$ surface tends to blur interference patterns more easily than for helium impact.

Something similar happens when we consider the wider channel $\langle\overline{1}, 0,1\rangle$, which displays an even stronger corrugation. In addition, this incidence direction does not run along a symmetry axis of the surface, and this results in angular distributions displaying asymmetric structures with respect to $\varphi_{f}=0$. Therefore, the high reactivity of $\mathrm{N}$ atoms favors the sensitivity of diffraction patterns to asymmetries across the channel, which in this case are originated from the contributions of target atoms from the second layer below the topmost atomic plane.

We have also analyzed the contribution of inelastic processes. Our results suggest that they might not be so important as expected in the considered energy range, which is in accord with available experimental results. Given its high resolution in energy, diffraction of fast atoms from surfaces may therefore become a useful quality check for both insulating and metallic PESs, also providing a promising tool for surface analysis.

\section{ACKNOWLEDGMENTS}

M.S.G. acknowledges financial support from CONICET, UBA, and ANPCyT of Argentina. G.A.B. acknowledges fruitful discussions with Dr. H. F. Busnengo regarding the PES calculation.
[1] H. Khemliche, P. Rousseau, P. Roncin, V. H. Etgens, and F. Finocchi, Appl. Phys. Lett. 95, 151901 (2009).

[2] A. Schüller, M. Busch, S. Wethekam, and H. Winter, Phys. Rev. Lett. 102, 017602 (2009).

[3] A. Schüller, H. Winter, M. S. Gravielle, J. M. Pruneda, and J. E. Miraglia, Phys. Rev. A 80, 062903 (2009).

[4] C. Díaz, P. Rivière, and F. Martín, Phys. Rev. Lett. 103, 013201 (2009).

[5] A. Schüller, S. Wethekam, and H. Winter, Phys. Rev. Lett. 98, 016103 (2007).

[6] P. Rousseau, H. Khemliche, A. G. Borisov, and P. Roncin, Phys. Rev. Lett. 98, 016104 (2007).

[7] A. Schüller and H. Winter, Phys. Rev. Lett. 100, 097602 (2008).

[8] A. Schüller, M. Busch, J. Seifert, S. Wethekam, H. Winter, and K. Gärtner, Phys. Rev. B 79, 235425 (2009).
[9] F. Aigner, N. Simonović, B. Solleder, L. Wirtz, and J. Burgdörfer, Phys. Rev. Lett. 101, 253201 (2008).

[10] M. S. Gravielle and J. E. Miraglia, Phys. Rev. A 78, 022901 (2008).

[11] M. S. Gravielle and J. E. Miraglia, Nucl. Instrum. Methods Phys. Res., Sect. B 267, 610 (2009).

[12] N. Bundaleski, H. Khemliche, P. Soulisse, and P. Roncin, Phys. Rev. Lett. 101, 177601 (2008).

[13] M. Busch, A. Schüller, S. Wethekam, and H. Winter, Surf. Sci. 603, L23 (2009).

[14] H. Khemliche, N. Bundaleski, P. Soulisse, and P. Roncin, Nucl. Instrum. Methods Phys. Res., Sect. B 267, 620 (2009).

[15] H. Ueta, M. A. Gleeson, and A. W. Kleyn, J. Phys. Chem. A 113, 15092 (2009).

[16] A. Groß, Surf. Sci. Rep. 32, 291 (1998). 
[17] A. Groß and M. Scheffler, Phys. Rev. B 57, 2493 (1998).

[18] G. J. Kroes, E. J. Baerends, and R. C. Mowrey, Phys. Rev. Lett. 78, 3583 (1997).

[19] M. Alducin, R. DíezMuiño, H. F. Busnengo, and A. Salin, J. Chem. Phys. 125, 144125 (2006).

[20] G. A. Bocan, R. DíezMuiño, M. Alducin, H. F. Busnengo, and A. Salin, J. Chem. Phys. 128, 154704 (2008).

[21] G. Kresse and J. Hafner, Phys. Rev. B 47, 558 (1993); 48, 13115 (1993); G. Kresse and J. Furthmüller, Comput. Mater. Sci. 6, 15 (1996); Phys. Rev. B 54, 11169 (1996).

[22] H. F. Busnengo, A. Salin, and W. Dong, J. Chem. Phys. 112, 7641 (2000).

[23] W. F. Avrin and R. P. Merrill, Surf. Sci. 311, 269 (1994).

[24] C. J. Joachain, Quantum Collision Theory (North-Holland, Amsterdam, 1979).

[25] J. P. Perdew, J. A. Chevary, S. H. Vosko, K. A. Jackson, M. R. Pederson, D. J. Singh, and C. Fiolhais, Phys. Rev. B 46, 6671 (1992).

[26] D. Vanderbilt, Phys. Rev. B 41, 7892 (1990).
[27] M. Methfessel and A. T. Paxton, Phys. Rev. B 40, 3616 (1989).

[28] M. V. Berry and K. E. Mount, Rep. Prog. Phys. 35, 315 (1972).

[29] The effective corrugation $\Delta z$ is defined as the normal distance between the average potentials along the row of $\mathrm{Ag}$ atoms and the middle of the channel.

[30] S. Lederer, K. Maass, D. Blauth, H. Winter, H. P. Winter, and F. Aumayr, Phys. Rev. B 67, 121405(R) (2003).

[31] J. I. Juaristi, F. J. García de Abajo, and P. M. Echenique, Phys. Rev. B 53, 13839 (1996).

[32] F. J. García de Abajo and P. M. Echenique, Phys. Rev. B 48, 13399 (1993).

[33] J. E. Miraglia and M. S. Gravielle, Phys. Rev. A 81, 042709 (2010).

[34] J. E. Valdes, P. Vargas, C. Celedon, E. Sanchez, L. Guillemot, and V. A. Esaulov, Phys. Rev. A 78, 032902 (2008).

[35] V. M. Silkin, M. Alducin, J. I. Juaristi, E. V. Chulkov, and P. M. Echenique, J. Phys. Condens. Matter 20, 304209 (2008). 